Article

\title{
Coupled coincidence and coupled common fixed points of a pair for mappings satisfying a weakly contraction type T-coupling in the context of quasi $\alpha b$-metric space
}

\author{
Kidane Koyas ${ }^{1}$ and Solomon Gebregiorgis ${ }^{1, *}$ \\ 1 Department of Mathematics, Jimma University, Jimma, Ethiopia. \\ * Correspondence: solomonggty@gmail.com
}

Received: 10 March 2020; Accepted: 30 September 2020; Published: 8 October 2020.

\begin{abstract}
In this paper, we have established a theorem involving a pair of mappings satisfying a weakly contraction type condition in the context of quasi $\alpha \mathrm{b}$-metric space and proved the existence and uniqueness of coupled coincidence and coupled common fixed points. The concept of weakly compatibility of the pair of maps is applied to show the uniqueness of coupled common fixed point. This work offers an extension to the published work of Nurwahyu and Aris [1].
\end{abstract}

Keywords: Coupled coincidence point, coupled common fixed point, Quasi $\alpha \mathrm{b}$-metric space, T-coupling, weakly contraction type.

MSC: 34B15, 34B18.

\section{Introduction}

$\mathbf{F}$ ixed point theory has been one of the most influential research topics in various fields of engineering and science. It is widely applied in solving linear algebraic equations, ordinary differential equations, integral equations, partial differential equations. The first most significant result of metric fixed point theory was given by the polish mathematician Stefan Banach, in 1922, which is known as Banach contraction principle. The famous Banach contraction principle states that in a complete metric space, a contraction self-map has a unique fixed point. It is one of the cornerstones in the development of nonlinear analysis.

The concept of b-metric spaces was introduced by Bakhtin [2] in 1989, who used it to prove a generalization of the Banach contraction principle in spaces endowed with such kind of metrics. Since then, this notion has been used by many authors to obtain various fixed point theorems. In 1993, Czewick [3] used b-metric space on his papers for their fixed point theorems on contraction mappings in the b-metric space. Then many authors also used the b-metric space for their fixed point theorems for several contraction mappings [4-8] and then other authors developed the b-metric space to become a quasi b-metric space [8,9]. The quasi b-metric space has been used on some weak contraction mappings, and the weak contraction mapping was introduced by [10]. The quasi $\alpha \mathrm{b}$-metric space was introduced by Nurwahyu [1]. It was developed from b-metric space by ignoring symmetry and modifying the triangular inequality condition of b-metric and they proposed and proved theorems which involve the existence and uniqueness of fixed point for weak contraction mappings in quasi $\alpha \mathrm{b}$-metric space.

The purpose of this study is to establish a theorem involving a pair of mappings satisfying a weakly contraction type T-coupling in the context of quasi $\alpha \mathrm{b}$-metric space and then prove the existence and uniqueness of coupled coincidence and coupled common fixed points. The concept of weakly compatibility of the pair of maps is applied to show the uniqueness of coupled common fixed point. This work is offers extension to the published work of Nurwahyu and Aris [1]. Finally, an illustrative example is presented to verify that all the conditions of the theorem are fulfilled.

\section{Preliminaries}

Now, we present relevant definitions and results that will be retrieved in the sequel.

Definition 1. [2] Let $X$ be a non-empty set and $b \geq 1$ be any given real number.

Let $d: X \times X \rightarrow[0, \infty)$ be a function satisfying the following conditions: 
(a) $d(x, y)=d(y, x)=0 \Leftrightarrow x=y$.

(b) $d(x, y)=d(y, x)$.

(c) $d(x, y) \leq b[d(x, z)+d(z, y)]$ for all $x, y, z \in X$.

Then $d$ is known as b-metric on $X$ and the pair $(X, d)$ is called a b-metric space.

Definition 2. [1] Let $X$ be a non-empty set and $0 \leq \alpha<1$ and $b \geq 1$ be a given real number. Let $d: X \times X \rightarrow$ $[0, \infty)$ be a function satisfying the the following conditions:

(a) $d(x, y)=d(y, x)=0 \Leftrightarrow x=y$.

(b) $d(x, y) \leq \alpha d(y, x)+\frac{1}{2} b[d(x, z)+d(z, y)]$ for all $x, y, z \in X$.

Then $d$ is known as quasi $\alpha \mathrm{b}$-metric on $X$ and the pair $(X, d)$ is called a quasi $\alpha \mathrm{b}$-metric space.

Definition 3. [12] Let $(X, d)$ be a quasi $\alpha \mathrm{b}$-metric space and $T: X \rightarrow X$ be a self-map, then $T$ is said to be a contraction mapping if there exists a constant $k \in[0,1)$ called a contraction factor, such that

$$
d(T x, T y) \leq k d(x, y)
$$

for all $x, y \in X$.

Definition 4. [1] Let $(X, d)$ be a quasi $\alpha$ b-metric space with $0 \leq \alpha<1$ and $b \geq 1$. A mapping $T: X \longmapsto X$ is called a weak contraction on $X$ if there exists a function $\varphi:[0, \infty) \longmapsto[0, \infty), \varphi(t)=0$ iff $t=0$ and satisfying the following condition:

$$
d(T x, T y) \leq d(x, y)-\delta \varphi(d(x, y))
$$

for all $x, y \in X$ where $0<\delta \leq 1$.

Definition 5. [1] A sequence $\left\{x_{n}\right\}$ in a quasi $\alpha$ b-metric space $(X, d)$ is said to converge to a point $x \in X$ if and only if

$$
\lim _{n \rightarrow \infty} d\left(x_{n}, x\right)=\lim _{n \rightarrow \infty} d\left(x, x_{n}\right)=0 .
$$

Definition 6. [1] A sequence $\left\{x_{n}\right\}$ in a quasi $\alpha$ b-metric space $(X, d)$ is called a Cauchy sequence if for every $\epsilon>0$, there exists a positive integer $n_{0}$ such that for $m, n>n_{0}$, we have $d\left(x_{n}, x_{m}\right)<\epsilon$. That is,

$$
\lim _{n, m \rightarrow \infty} d\left(x_{n}, x_{m}\right)=\lim _{n, m \rightarrow \infty} d\left(x_{m}, x_{n}\right)=0 .
$$

Definition 7. [1] A quasi $\alpha \mathrm{b}$-metric space is called complete if every Cauchy sequence converges to an element in the same metric space.

Definition 8. [12] Let $X$ be a nonempty set and $T: X \rightarrow X$ a self-map. We say that $x$ is a fixed point of $T$ if $T x=x$.

Definition 9. [13] An element $(x, y) \in X \times X$, where $X$ is any non-empty set, is called a coupled fixed point of the mapping $F: X \times X \rightarrow X$ if $F(x, y)=x$ and $F(y, x)=y$.

Definition 10. [14]. Let $(X, d)$ be a quasi $\alpha \mathrm{b}$-metric space and $A$ and $B$ be two non-empty subsets of $X$. Then a function $F: X \times X \rightarrow X$ is said to be a coupling with respect to $A$ and $B$ if $F(x, y) \in B$ and $F(y, x) \in A$ where $x \in A$ and $y \in B$.

Definition 11. [15]. Let $A$ and $B$ be any two non-empty subsets of a quasi $\alpha \mathrm{b}$-metric space $(X, d)$ and $T: X \rightarrow X$ be a self-map on $X$. Then $T$ is said to be SCC-Map with respect to $A$ and $B$ ), if

(a) $T(A) \subseteq A$ and $T(B) \subseteq B$,

(b) $T(A)$ and $T(B)$ are closed in $X$.

Definition 12. [16] An element $(x, y) \in X \times X$ is called a coupled coincidence point of the mappings $F$ : $X \times X \rightarrow X$ and $g: X \rightarrow X$ if $F(x, y)=g(x)$ and $F(y, x)=g(y)$, and $(g x, g y)$ is called coupled point of coincidence. 
Definition 13. [16] An element $(x, y) \in X \times X$, where $X$ is any non-empty set, is called a coupled common fixed point of the mappings $F: X \times X \rightarrow X$ and and $g: X \rightarrow X$ if $F(x, y)=g(x)=x$ and $F(y, x)=g(y)=y$.

Definition 14. [16] The mappings $F: X \times X \rightarrow X$ and $g: X \rightarrow X$ are called weakly Compatible if $g(F(x, y))=$ $F(g x, g y)$ and $g(F(y, x))=F(g y, g x)$ whenever $g x=F(x, y)$ and $g y=F(y, x)$.

Definition 15. A function $\omega:[0, \infty) \rightarrow[0, \infty)$ is called an altering distance function, if the following properties are satisfied:

(a) $\omega$ is monotonically non-deceasing and continuous.

(b) $\omega(t)=0$ if and only if $t=0$.

Theorem 1. [1] Let $(X, d)$ be a complete quasi $\alpha b$-metric space with $0 \leq \alpha<1$ and $b \geq 1$. Let $F: X \rightarrow X$ be a self-map satisfying the following condition:

$$
d(F x, F y) \leq \min \{d(x, F x), d(F y, y)\}-k \cdot \omega(\max \{d(x, F x), d(F y, y)\})
$$

for all $x, y \in X$ and $k>0, \omega:[0, \infty) \rightarrow[0, \infty)$ is a continuous function and $\omega(t)=0$ iff $t=0$. Then $F$ has a unique fixed point in $X$.

\section{Main results}

At this stage, we state our theorem and come up with the main findings.

Theorem 2. Let $A$ and $B$ be any two non-empty closed subsets of a complete quasi $\alpha b$-metric space $(X, d)$ with $0 \leq \alpha<1$ ,$b \geq 1$, and $k>0$. Let $T: X \rightarrow X$ is SCC-Map on $X$ (with respect to $A$ and $B$ ) and $F: X \times X \rightarrow X$ be $a$ T-coupling (with respect to $A$ and $B$ ) if there exists an altering distance function $\omega$ such that

$$
d[F(x, y), F(u, v)] \leq \min \{d(T x, F(x, y)), d(F(v, u), T v)\}-k \cdot \omega(\max \{d(T x, F(x, y)), d(F(v, u), T v)\})(1)
$$

for any $x, v \in A$ and $y, u \in B$, then

(i) $F(X \times X) \subseteq T(X)$

(ii) $T(A) \cap T(B) \neq \varnothing$

(iii) $T$ and $F$ have a coupled coincidence point in $A \times B$.

(iv) If $T$ and $F$ are weakly compatible, then $T$ and $F$ have a unique coupled common fixed point in $A \times B$.

Proof. Since $A$ and $B$ are non-empty subsets of $X$ and $T$ is a type-T coupling with respect to $A$ and $B$, then for $x_{0} \in A$ and $y_{0} \in B$, we define the sequences $\left\{x_{n}\right\}$ and $\left\{y_{n}\right\}$ in $A$ and $B$ respectively such that

$$
T x_{1}=F\left(x_{0}, y_{0}\right) \text { and } T y_{1}=F\left(y_{0}, x_{0}\right) .
$$

This can be done because $F(X \times X) \subseteq T(X)$. Continuing this process, we can construct two sequences $\left\{x_{n}\right\}$ and $\left\{y_{n}\right\}$ in $X$ such that

$$
T x_{n+1}=F\left(x_{n}, y_{n}\right) \text { and } T y_{n+1}=F\left(y_{n}, x_{n}\right)
$$

and then, we have $d\left(T x_{n}, T x_{n+1}\right)=d\left[F\left(x_{n-1}, y_{n-1}\right), F\left(x_{n}, y_{n}\right)\right]$. Using equations (1) and (2), we have

$$
\begin{aligned}
d\left(T x_{n}, T x_{n+1}\right)= & d\left[F\left(x_{n-1}, y_{n-1}\right), F\left(x_{n}, y_{n}\right)\right] \\
\leq & \min \left\{d\left(T x_{n-1}, F\left(x_{n-1}, y_{n-1}\right)\right), d\left(F\left(y_{n}, x_{n}\right), T y_{n}\right)\right\} \\
& -k \cdot w\left(\max \left\{d\left(T x_{n-1}, F\left(x_{n-1}, y_{n-1}\right)\right), d\left(F\left(y_{n}, x_{n}\right), T y_{n}\right)\right\}\right) \\
\leq & \min \left\{d\left(T x_{n-1}, T\left(x_{n-1}, y_{n-1}\right)\right), d\left(F\left(y_{n}, x_{n}\right), T y_{n}\right)\right\} \\
\leq & d\left(T x_{n-1}, F x_{n}\right) .
\end{aligned}
$$

Thus, we have a non-negative and non-increasing sequence $\left\{T x_{n}\right\}$. Therefore, there exists $L \geq 0$ such that $\lim _{n \rightarrow \infty} d\left(T x_{n}, T x_{n+1}\right)=L$. Since $\omega$ is continuous on $[0, \infty)$ and using (3) and for $n \rightarrow \infty$, we get $L \leq L-K \cdot \omega(L)$. 
It follows that, $\omega(L)=0$ which in turn implies that $L=0$ since $\omega$ is an altering distance function. Similarly, we can obtain that $\lim _{n \rightarrow \infty} d\left(T y_{n}, T y_{n+1}\right)=0$.

Now, we show that $\left\{T x_{n}\right\}$ and $\left\{T y_{n}\right\}$ are Cauchy sequences in $T(X)$. Let $m>n \geq 1$ and using Equations (1) and (2), we have

$$
\begin{aligned}
d\left(T x_{n}, T x_{m}\right)= & d\left[F\left(x_{n-1}, y_{n-1}\right), F\left(x_{m-1}, y_{m-1}\right)\right] \\
\leq & \min \left\{d\left(T x_{n-1}, F\left(x_{n-1}, y_{n-1}\right)\right), d\left(F\left(y_{m-1}, x_{m-1}\right), T y_{m-1}\right)\right\} \\
& -k \cdot w\left(\max \left\{d\left(T x_{n-1}, F\left(x_{n-1}, y_{n-1}\right)\right), d\left(F\left(y_{m-1}, x_{m-1}\right), T y_{m-1}\right)\right\}\right) \\
\leq & \min \left\{d\left(T x_{n-1}, T x_{n}\right), d\left(T x_{m}, T y_{m-1}\right)\right\} \\
\leq & d\left(T x_{n-1}, T x_{n}\right) .
\end{aligned}
$$

Similarly, we can show that

$$
d\left(T x_{m}, T x_{n}\right) \leq d\left(T x_{m-1}, T x_{m}\right)
$$

Taking Equations (4) and (5) as $n, m \rightarrow \infty$, we get $d\left(T x_{n}, T x_{m}\right) \rightarrow 0$ and $d\left(T x_{m}, T x_{n}\right) \rightarrow 0$. Following the same procedure as above, we can show that $d\left(T y_{n}, T y_{m}\right) \rightarrow 0$ and $d\left(T y_{m}, T y_{n}\right) \rightarrow 0$. Hence $\left\{T x_{n}\right\}$ and $\left\{T y_{n}\right\}$ are Cauchy sequences in $T(A)$ and $T(B)$ respectively. Since $T(A)$ and $T(B)$ are closed subset of a complete quasi $\alpha \mathrm{b}$-metric space $X$, we conclude that $\left\{T x_{n}\right\}$ and $\left\{T y_{n}\right\}$ are convergent in $T(A)$ and $T(B)$ respectively. Thus, there exist $r \in T(A)$ and $s \in T(B)$ such that

$$
T x_{n} \rightarrow r \text { and } T y_{n} \rightarrow s \text { as } n \rightarrow \infty .
$$

Using Equations (1) and (2), we have

$$
\begin{aligned}
d\left(T x_{n}, T y_{n}\right)= & d\left[F\left(x_{n-1}, y_{n-1}\right), F\left(y_{n-1}, x_{n-1}\right)\right] \\
\leq & \min \left\{d\left(T x_{n-1}, F\left(x_{n-1}, y_{n-1}\right)\right), d\left(F\left(x_{n-1}, y_{n-1}\right), T x_{n-1}\right)\right\} \\
& -k \cdot w\left(\max \left\{d\left(T x_{n-1}, F\left(x_{n-1}, y_{n-1}\right)\right), d\left(F\left(x_{n-1}, y_{n-1}\right), T x_{n-1}\right)\right\}\right) \\
\leq & \min \left\{d\left(T x_{n-1}, F\left(x_{n-1}, y_{n-1}\right)\right), d\left(F\left(x_{n-1}, y_{n-1}\right), T x_{n-1}\right)\right\} \\
\leq & d\left(T x_{n-1}, T x_{n}\right) .
\end{aligned}
$$

Similarly, we can show that

$$
d\left(T y_{n}, T x_{n}\right) \leq d\left(T y_{n-1}, T y_{n}\right) .
$$

Taking Equations (7) and (8) as $n, m \rightarrow \infty$, we get

$$
d\left(T x_{n}, T y_{n}\right) \rightarrow 0 \text { and } d\left(T y_{n}, T x_{n}\right) \rightarrow 0 .
$$

Therefore, from (6) and (9), we have

$$
s=r .
$$

As $r \in T(A)$ and $s \in T(B) \Rightarrow s=r \in T(A) \cap T(B)$. This proves Part (i), i.e., $T(A) \cap T(B) \neq \varnothing$. Now, since $r \in T(A)$ and $s \in T(B)$, there exist $x \in A$ and $y \in B$ such that $r=T(x)$ and $s=T(y)$. From Equations (6) and (10) we have

$$
\begin{aligned}
T x_{n} \rightarrow T(x), T y_{n} & \rightarrow T(y) \\
T(x) & =T(y) .
\end{aligned}
$$

Using Definition 2, we have

$$
d(r, F(x, y)) \leq \alpha d(F(x, y), r)+\frac{1}{2} b\left[d\left(r, T x_{n}\right)+d\left(T x_{n}, F(x, y)\right)\right]
$$

and

$$
d(F(x, y), r) \leq \alpha d(r, F(x, y))+\frac{1}{2} b\left[d\left(T x_{n}, r\right)+d\left(F(x, y), T x_{n}\right)\right]
$$


Applying (1) and (2) in (13) and (14) and taking their limits as $n \rightarrow \infty$, we get

$$
d(r, F(x, y)) \leq \alpha d(F(x, y), r)
$$

and

$$
d(F(x, y), r) \leq \alpha d(r, F(x, y))
$$

Substituting (16) into (15), we get

$$
\left(1-\alpha^{2}\right) d(r, F(x, y)) \leq 0 .
$$

This is only possible if $d(r, F(x, y))=0$ since $0 \leq \alpha<1$. Similarly, we can show that $d(F(x, y), r)=0$. Hence $F(x, y)=r$. Moreover, we can show that $F(y, x)=s$. Hence, $(T x, T y)$ is coupled point of coincedence of $T$ and $F$. Now, we claim that $(T x, T y)$ is the unique coupled point of coincidence of $T$ and $F$. Suppose not. So, we have another coupled point of coincedence say $\left(T x^{*}, T y^{*}\right)$ where $\left(x^{*}, y^{*}\right) \in X^{2}$ with $T x^{*}=F\left(x^{*}, y^{*}\right)$ and $T y^{*}=F\left(y^{*}, x^{*}\right)$. Using Equations (1) and (2), we have

$$
\begin{aligned}
d(T x, T x) & =d[F(x, y), F(x, y)] \\
& \leq \min \{d(T x, F(x, y)), d(F(y, x), g y)\}-k \cdot w(\max \{d(T x, F(x, y)), d(F(y, x), T y)\}) \\
& =\min \{d(T x, T x), d(T y, T y)\}-k \cdot w(\max \{d(T x, T x), d(T y, T y)\}) \\
& \leq \min \{d(T x, T x), d(T y, T y)\}-k \cdot w(\max \{d(T x, T x), d(T y, T y)\}) .
\end{aligned}
$$

From (18), it follows that $d(T x, T x) \leq d(T x, T x)-k \cdot w(\max \{d(T x, T x), d(T y, T y)\})$. Hence $d(T x, T x)=$ 0 and $d(T y, T y)=0$. Now, let us consider $d\left(T x, T x^{*}\right)$. Using Equations (1) and (2), we have

$$
\begin{aligned}
d\left(T x, T x^{*}\right) & =d\left[F(x, y), F\left(x^{*}, y^{*}\right)\right] \\
& \leq \min \left\{d(T x, F(x, y)), d\left(F\left(y^{*}, x^{*}\right), T y^{*}\right)\right\}-k \cdot w\left(\max \left\{d(T x, F(x, y)), d\left(F\left(y^{*}, x^{*}\right), t y^{*}\right)\right\}\right) \\
& =\min \left\{d(T x, T x), d\left(T y^{*}, T y^{*}\right)\right\}-k \cdot w\left(\max \left\{d(T x, T x), d\left(T y^{*}, T y^{*}\right)\right\}\right) .
\end{aligned}
$$

From (19), it follows that $d\left(T x, T x^{*}\right) \leq 0$. Hence $d\left(T x, T x^{*}\right)=0$. Again, using Equations (1) and (2), we have

$$
\begin{aligned}
d\left(T x^{*}, T x\right) & =d\left[F\left(x^{*}, y^{*}\right), F(x, y)\right] \\
& \leq \min \left\{d\left(T x^{*}, F\left(x^{*}, y^{*}\right)\right), d(F(y, x), T y)\right\}-k \cdot w\left(\max \left\{d\left(T x^{*}, F\left(x^{*}, y^{*}\right)\right), d(F(y, x), T y)\right\}\right) \\
& =\min \left\{d\left(T x^{*}, T x^{*}\right), d(T y, T y)\right\}-k \cdot w\left(\max \left\{d\left(T x^{*}, T x^{*}\right), d(T y, T y)\right\}\right) .
\end{aligned}
$$

From (20), it follows that $d\left(T x^{*}, T x\right) \leq 0$. Hence $d\left(T x^{*}, T x\right)=0$. From Equations (19) and (20), we deduce that $T x=T x^{*}$. Similarly, we can show that $T y=T y^{*}$. Therefore, we have a unique coupled point of coincidence.

Now, we show that $T$ and $F$ have coupled common fixed point. In order to do that, we consider $d(T x, F(x, x))$. Using (1), we have

$$
\begin{aligned}
d(T x, F(x, x)) & =d[F(x, y), F(x, x)] \\
& \leq \min \{d(T x, F(x, y)), d(F(x, x), T x)\}-k \cdot w(\max \{d(T x, F(x, y)), d(F(x, x), T x)\}) \\
& =\min \{d(T x, T x), d(F(x, x), T x)\}-k \cdot w(\max \{d(T x, T x), d(F(x, x), T x)\}) \\
& \leq \min \{0, d(F(x, x), T x)\}-k \cdot w(\max \{0, d(F(x, x), T x)\}) .
\end{aligned}
$$

From (21), it follows that

$$
d(T x, F(x, x)) \leq-k \cdot w(d(F(x, x), T x))
$$


From (22), we can deduce that $d(F(x, x), T x)=0$ and $d(T x, F(x, x))=0$. Hence $T x=F(x, x)$. Now, let $T x=u$, then we have that $u=T x=F(x, x)$. Since $T$ and $F$ are weakly compatible, we have $T u=T(T x)=$ $T(F(x, x))=F(T x, T x)=F(u, u)$. Hence $(T u, T u)$ is a coupled point of coincidence and $(u, u)$ is a coupled coincidence point of $T$ and $F$. The uniqueness of coupled point of coincidence implies that $T u=u=T x$. Therefore $F(u, u)=T u=u$.That is $(u, u)$ is the coupled common fixed point of $T$ and $F$. Finally, we show the uniqueness of a coupled common fixed point of $T$ and $F$. let $\left(u^{*}, u^{*}\right) \in X^{2}$ be another coupled common fixed point of $F$ and $T$. That is, $u^{*}=T u^{*}=F\left(u^{*}, u^{*}\right)$. Hence $(T u, T u)$ and $\left(T u^{*}, T u^{*}\right)$ are two coupled points of coincidence of $T$ and $F$. The uniqueness of coupled point of coincidence implies that $T u=T u^{*}$ and so $F\left(u^{*}, u^{*}\right)=u^{*}=u$. Hence $(u, u)$ is the unique coupled common fixed point of $T$ and $F$.

Remark 1. If we take $T=I$ (the identity map) and change the mapping $F: X \times X \rightarrow X$ to $F: X \rightarrow X$, then Theorem 2 will reduce to Theorem 1 of Nurwahyu and Aris [1].

Example 1. Let $X=[0,5]$ which is defined by $d(x, y)=|x-y|$ and $A=\{1\}$ and $B=\{1,2\}$. Then $A$ and $B$ are closed subsets of $X$. We define $F: X \times X \rightarrow X$ by $F(x, y)=\min \{x, y\}$, for all $x, y \in X$. Let $T: X \rightarrow X$ be defined by

$$
T(x)= \begin{cases}1 & \text { if } 0 \leq x<2 \\ 2 & \text { if } 2 \leq x \leq 5\end{cases}
$$

Also, we define $\omega:[0, \infty) \rightarrow[0, \infty)$ by $\omega(t)=t^{2}$. Then, clearly $\omega$ is altering distances function. $T(A)=$ $\{1\}$ and $T(B)=\{1,2\}$. So, $T(A)$ and $T(B)$ are closed subsets of a complete quasi $\alpha \mathrm{b}$-metric space $X=[0,5]$. Hence $T: X \rightarrow X$ is a SCC-Map. Now, we show that $T$ is $F$-coupling with respect to $A$ and $B$ as $T(A) \cap B=\{1\}$ and $T(B) \cap A=\{1\}$. So, for all $x \in A$ and $y \in B$, we have $F(x, y)=1 \in B$ and $F(y, x)=1 \in A$, i.e., $F(x, y) \in T(A) \cap B$ and $F(y, x) \in T(B) \cap A$ which show that $F$ is a $T$-coupling with respect to $A$ and $B$. Now, it remains to prove that $\mathrm{F}$ is a contractive $T$-coupling w.r.t. $A$ and $B$. Let $x, v \in A$ and $y, u \in B$ i.e., $x=1$ and $y=1,2$. Four cases will arise for $y$ and $u$.

Case (i): $x=v=1$ and $y=u=1$.

Case (ii): $x=v=1$ and $y=1, u=2$.

Case (iii): $x=v=1$ and $y=2, u=1$.

Case (iv): $x=v=1$ and $y=u=2$.

For Case (i), i.e., $x=v=1$ and $y=u=1$, we have $F(x, y)=F(u, v)=F(v, u)=F(1,1)=1$, $T(x)=T(v)=T(1)=1, d(1,1)=0$, and

$$
\begin{aligned}
d[F(x, y), F(u, v)] & \leq \min \{d(T x, F(x, y)), d(F(v, u), T v)\}-k \cdot \omega(\max \{d(T x, F(x, y)), d(F(v, u), T v)\}), \\
d(0) & \leq 0-k \cdot \omega(0), \\
0 & \leq 0
\end{aligned}
$$

which proves Case (i).

In a similar fashion, we can show for the other three cases. Hence, $T$ and $F$ satisfy all the conditions of Theorem 2. Thus $T$ and $F$ have a strong coupled fixed points in $A \cap B$. Clearly $T(A) \cap T(B)=\{1\} \neq \varnothing .1$ is the unique strong coupled coincidence point and $(1,1)$ is the unique coupled common fixed point of $\mathrm{T}$ and $\mathrm{g}$ in $A \cap B$ as $T(1)=F(1,1)=\min \{1,1\}=1$.

\section{Conclusion}

In this paper, we have established a theorem involving a pair of mappings satisfying a weakly contraction type T-coupling in the context of quasi $\alpha \mathrm{b}$-metric space and then prove the existence and uniqueness of coupled coincidence and coupled common fixed points. The concept of weakly compatibility of the pair of maps is applied to show the uniqueness of coupled common fixed point. We also provide an example in support of our main result. Our work extended the published work of Nurwahyu and Aris [1].

Acknowledgments: The authors want to thank the anonymous referee for the throughout reading of the manuscript and several suggestions that help us improve the presentation of the paper. We would also like to thank the College of Natural Sciences, Jimma University for funding this staff research project.

Author Contributions: All authors contributed equally to the writing of this paper. All authors read and approved the final manuscript. 
Conflicts of Interest: "The authors declare no conflict of interest."

\section{References}

[1] Nurwahyu, B. \& Aris, N. (2018). Fixed point theorems on some weak contraction mappings in quasi $\alpha$ b-metric space. In Journal of Physics: Conference Series, volume 1013, (pp. 012151). IOP Publishing.

[2] Bakhtin, I. A. (1989). The contraction mapping principle in quasimetric spaces. Functional Analysis, 30, 26-37.

[3] Czerwik, S. (1993). Contraction mappings in b-metric spaces. Acta Mathematica Universitatis Ostraviensis, 1, 5-11.

[4] Ansari, A. H., Chandok, S., \& Ionescu, C. (2014). Fixed point theorems on b-metric spaces for weak contractions with auxiliary functions. Journal of Inequalities and Applications, 2014(1), 429.

[5] Joseph, J. M., Roselin, D. D., \& Marudai, M. (2014). Fixed point theorems on multi valued mappings in b-metric spaces. SpringerPlus, 5(1), 217.

[6] Kamran, T., Samreen, M., \& UL Ain, Q. (2017). A generalization of b-metric space and some fixed point theorems. Mathematics, 5(2), 19.

[7] Mishra, P. K .and Sachdeva, S. \& Banerjee, S. K. (2014). Some fixed point theorems in b-metric space. Turkish Journal of Analysis and Number Theory, 2(1), 19-22.

[8] Shatanawi, W., Pitea, A., \& Lazovic, R. (2014). Contraction conditions using comparison functions on b-metric spaces fixed point theory and applications. Fixed Point Theory and Applications, 2014(1), 135.

[9] Klin-eam, C. \& Suanoom, C. (2015). Dislocated quasi-b-metric spaces and fixed point theorems for cyclic contractions. Fixed Point Theory and Applications, 2015(1), 174.

[10] Alber, Y. I., \& Guerre-Delabriere, S. (1997). Principle of weakly contractive maps in Hilbert spaces. In New results in operator theory and its applications (pp. 7-22). Birkhäuser, Basel.

[11] Nurwahyu, B. (2017). Fixed point theorems for generalized contraction mappings in quasi $\alpha$ b-metric space. Far East Journal of Mathematical Sciences, 102(9), 2105-2119.

[12] Banach, S. (1922). Sur les opérations dans les ensembles abstraits et leur application aux équations intégrales. Fundamenta Mathematicae, 3(1), 133-181.

[13] Bhaskar, T. G. \& Lakshmikantham, V. (2006). Fixed point theorems in partially ordered metric spaces and applications. Nonlinear Analysis: Theory, Methods \& Applications, 65(7), 1379-1393.

[14] Choudhury, B. S., Maity, P., \& Konar, P. (2017). Fixed point results for couplings on metric spaces. UPB Scientific Bulletin, Series A: Applied Mathematics and Physics, 79(1), 1-12.

[15] Khan, M., Swaleh, M., \& Sessa, S. (1984). Fixed point theorems by altering distances between the points. Bulletin of the Australian Mathematical Society, 30(1), 1-9.

[16] Lakshmikantham, V. \& Ciric, L. (2019). Coupled coincidence point theorems for nonlinear contractions in partially ordered metric spaces. Nonlinear Analysis, 70(1), 4341-4349. 
(c) 2020 by the authors; licensee PSRP, Lahore, Pakistan. This article is an open access article distributed under the terms and conditions of the Creative Commons Attribution (CC-BY) license (http://creativecommons.org/licenses/by/4.0/). 